\title{
Enjeux de la formation au numérique des communautés scolaires dans une perspective de transformation écosociale
}

\section{Challenges of digital education for a school community that contributes}

\author{
to eco-social transformation
}

\author{
Aurèlie Zwang ${ }^{1}$ \\ ${ }^{1}$ Muséum National d'Histoire Naturelle de Paris, aurelie.zwang@mnhn.fr
}

RÉSUMÉ. Avec un positionnement hybride entre théorie et pratique, cet article prend appui sur des expériences du groupe d'intégration pédagogique de l'information et de la communication (GIPTIC) en éducation au développement durable (EDD) de l'académie de Paris pour émettre des propositions de formation de la communauté éducative à l'usage des technologies de l'information et de la communication en vue d'une transformation écosociale : privilégier une approche culturelle et pédago-centrée des TIC, identifier la diversité des TIC pour en estimer les possibilités et les limites selon ses propres objectifs éducatifs, initier aux TIC pouvant soutenir des démarches pédagogiques de coopération, de mise en débat, de formation à l'esprit critique et à la notion de communs, et enfin ne pas occulter la place des TIC euxmêmes dans la crise socio-écologique actuelle.

ABSTRACT. With a hybrid positioning between theory and practice, this article is based on the experiences of the Group for the Pedagogical Integration of Information and Communication (GIPTIC) in Education for Sustainable Development (ESD) of the Paris Academy to make proposals for forming the educational community to use information and communication technologies for an eco-social transformation: favour a cultural and pedagogical-centred approach to ICTs, identify the diversity of ICTs in order to assess their possibilities and limitations according to its own objectives, introduce ICTs that can support educational approaches of cooperation, debate, training in critical thinking and the notion of commonality, and finally do not overshadow the place of ICTs themselves in the current socio-ecological crisis.

MOTS-CLÉS. Numérique, Education au développement durable, Transformation éco-sociale, Formation des enseignants, Praticien réflexif.

KEYWORDS. Digital, Education for sustainable development, Eco-social transformation, Teacher training, Reflection practitioner.

Cet article est issu d'une communication réalisée lors du colloque «changements et transitions », lequel proposait dans son organisation la rencontre entre chercheurs et acteurs dits de «terrain » (enseignants, formateurs, membres d'associations). Il inscrivait donc dans ses fondements la possibilité de ponts théoriques, méthodologiques et pratiques entre différents milieux contribuant à l'éducation aux problématiques socio-environnementales. Au-delà des spécificités professionnelles, une convergence s'est dessinée: celle de la nécessité d'une éducation en vue d'une transformation écosociale. C'est probablement sur et pour cette visée partagée qu'a émergé lors du colloque la notion d'« acteur hybride », à la jonction entre différents champs professionnels, tel le « marginal sécant » que Michel Crozier et Erhard Friedberg envisagent comme « un acteur [...] partie prenante dans plusieurs systèmes d'action en relation les uns avec les autres et qui peut, de fait, peut jouer le rôle indispensable d'intermédiaire et d'interprète entre des logiques d'actions différentes, voire contradictoires. » (Crozier et Friedberg, 2014, p. 86). Les «acteurs hybrides » seraient ces passeurs entre différents mondes de l'éducation (recherche et enseignement scolaire, enseignement scolaire et animation, formation et enseignement universitaire, etc.) qui, agissant encore trop souvent parallèlement, gagneraient sans aucun doute à unir leurs compétences pour cette finalité commune.

«Acteur hybride» de fait, car chercheuse en éducation à l'environnement et au développement durable (désormais EDD) mais aussi formatrice et enseignante menant des expérimentations 
pédagogiques depuis 15 ans, je propose ici une analyse qui, dès son intention, se situait entre le «terrain » et ce que celui-ci a déclenché comme nécessité de recours à des éléments théoriques. En somme, le lecteur voudra bien accepter de lire un texte situé dans un espace intermédiaire entre empirisme et conceptualisation, soit une forme de «composite», au sens donné par Joëlle le Marec (2002) : «le concept d'une part, le terrain de l'autre, doivent se contraindre l'un l'autre, ils doivent se contraindre à se transformer mutuellement pour aider à se rapprocher de ce qu'on entrevoit et qui est toujours un déséquilibre à résoudre entre ce que l'on voudrait penser et ce que l'on saisit empiriquement. » (2002, paragraphe 65).

On y trouvera donc des raisonnements en construction, nourris de mes problématiques de recherche sur les formes matérielles et symboliques de médiation des savoirs, des valeurs et des représentations relatifs aux problématiques socio-environnementales dans l'École (dont(Zwang, 2016), et de mes pratiques de formation dans un domaine en émergence dans la francophonie (Girault, 2017): l'intégration des technologies de l'information et de la communication (désormais TIC) en EDD. Dans un aller-retour permanent entre « terrain » et théorie, ce texte propose de dégager quelques propositions pour la formation au «numérique » en EDD, en axant plus particulièrement l'analyse sur les éléments qui pourraient contribuer à une transformation écosociale. Le «terrain » dont il est question concerne à la fois des pratiques de classe et des pratiques de formation en lien avec un groupe particulier de l'académie de Paris : le groupe d'intégration pédagogique des technologies de l'information et de la communication en éducation au développement durable (désormais GIPTIC EDD).

\section{Le GIPTIC EDD de l'académie de Paris : un groupe à positionnement singulier}

\subsection{Contexte institutionnel}

Sous le slogan de 2012 «L'école change avec le numérique», les «outils et médias éducatifs numériques » (Mœglin, 2015) se voient largement légitimés sous la forme d'un « grand plan numérique pour l'éducation » dont les moyens se sont traduits par le développement de collèges « connectés », d'appels à projet nationaux et de formations. Depuis 2013, l'académie de Paris « met [donc] en œuvre la politique du numérique éducatif ${ }^{1}$ via un service dédié : la Délégation académique au numérique éducatif (DANE) avec pour mission officielle de « conduire le changement », sans toutefois préciser la nature exacte de celui-ci. Pour ce faire, 16 GIPTIC ont pour rôle «d'inspirer», «de former» et «d'accompagner » (Taillard, 2017) les enseignants - le plus souvent sur la base du volontariat - selon un plan de travail commun.

Le GIPTIC EDD a été créé en 2015 sous l'impulsion des inspecteurs coordonnateurs académiques. Il est le seul de l'académie de Paris qui ne soit pas strictement adossé à une discipline. Nous ${ }^{2}$ sommes une petite équipe d'enseignants et de formateurs pluridisciplinaires : technologie, sciences physiquechimie, sciences de la vie et de la Terre, sciences biologiques et sociale appliquée et potentiellement histoire-géographie et sciences économiques et sociales ${ }^{3}$. Nous nous emparons du cahier des charges institutionnel comme levier de développement des logiques d'analyse, de réflexivité et de collaboration par et pour des tâches et/ou des projets d'établissement construit(e)s autour de problématiques socioécologiques, et ceci selon plusieurs partis pris.

\footnotetext{
${ }^{1}$ Source : https://www.ac-paris.fr/portail/jcms/p2 1054228/delegation-academique-au-numerique-educatif-dane-paris, consultée le 14 janvier 2018.

${ }^{2}$ Le « nous » de cette communication correspond au groupe de formateurs.

${ }^{3}$ Ont été ou sont membres du GIPTIC EDD : Julien Chamboredon, professeur de SVT, Alexandre Ligneau, professeur de sciences biologiques et sociale, Vincent Muracciole, professeur de technologie, Ludovic Réby, professeur de sciences physique-chimie. Nous remercions aussi Émilie Giniès, professeur d’histoire géographie, pour ses échanges fructueux avec le groupe.
} 


\subsection{Partis pris du GIPTIC EDD}

Notre premier parti pris est d'ordre épistémologique. Cette réflexion a mûri progressivement car, à l'instar d'autres acteurs de «terrain », nous n'avons pas d'emblée interrogé la nature des objets à intégrer pour une mise en œuvre - différente ? facilitée ? limitée ? - de l'EDD. Baignés dans le discours institutionnel dominant qui fait potentiellement du numérique le moteur de « la plus grande révolution pédagogique de l'histoire » (Fourgous, 2012, p. 125), la prise de recul s'est structurée autour du paradoxe qu'il est attendu de l'usage d' «outils » soit, au sens strict, des prothèses ou de simples prolongements de nos activités, une transformation majeure de l'école. Or selon Éric Bruillard (Bruillard, 2012) : «si l'idée d'outil semble satisfaire tout le monde, elle est incomplète voire fausse. L'ordinateur et toutes les technologies informatiques ne sont pas seulement des effecteurs, des aides à l'action, mais aussi des instruments, qui nous donnent à voir le « monde » et participent à notre propre construction de ce monde. ». Mais au-delà de la conception instrumentale des TIC, toute leur portée communicationnelle est à considérer, ce qui amène à les définir comme des « médias informatisés » (Jeanneret, 2011). Conformément au positionnement hybride introduit ci-dessus, j'emploierai encore ici le vocable associé à la notion «d'instruments » pour respecter le cheminement épistémologique du groupe, même si dans mes travaux plus récents (Zwang, 2019), issus d'analyses de séances pédagogiques menées par le GIPTIC EDD, je pense désormais davantage l'opérativité des TIC dans leur dimension communicationnelle. En bref, si le groupe ne considère pas les TIC comme des remèdes miracles en pédagogie, il ne les appréhende pas non plus comme les objets neutres que la dénomination d' « outil » sous-entend, même malgré elle.

Notre deuxième parti-pris est d'ordre pédagogique. Nous croyons en l'éducation comme creuset d'une transformation écosociale par le déploiement d'une écocitoyenneté critique reposant sur le développement de compétences critiques, éthiques et politiques (Sauvé, 2013). La pédagogie que nous essayons in fine d'impulser s'inspire des principes suivants : " on apprend la démocratie en vivant la démocratie, on apprend l'éthique en intégrant une dimension d'éthique aux situations d'apprentissage, on apprend le rapport à l'environnement en s'immergeant dans les réalités » (Sauvé, 2013, p. 31). Notre crédo, qui s'appuie sur des expérimentations menées en classe, est donc de former les membres des communautés scolaires à une intégration des TIC en EDD pour faire vivre aux élèves des situations éducatives où ces compétences sont, autant que possible, mobilisées.

Notre troisième parti pris, d'ordre andragogique, est celui de la promotion et la mise en œuvre des logiques de co-formation entre les adultes. En effet, le GIPTIC EDD a deux spécificités : l'interdisciplinarité et l'intercatégorialité. Il s'adresse aux enseignants de toutes disciplines ainsi qu'aux autres membres des communautés scolaires: des chefs d'établissements, des conseillers principaux d'éducation et des personnels technique et administratif participent aux formations, souvent en tant que référents de leur établissement. En plus de l'apport de contenus et des temps dédiés à la manipulation des technologies, une part importante est laissée aux échanges (Olry-Louis, 2003) pour ouvrir sur la multiplicité des appropriations à travers des retours d'expériences ou des points de vue professionnels différents. Ce principe, fondé sur une activité dialogique (Olry-Louis et Soidet, 2003), est en premier lieu appliqué au GIPTIC lui-même car nos origines disciplinaires modèlent différentes didactiques ou démarches pédagogiques comme autant d'obstacles potentiels malgré nos visées communes. Une progressive acculturation mutuelle a lieu autant au cours de réunions institutionnalisées que d'échanges informels (Gilbert, 2018) et elle est réellement effective lors de la conception des formations et des projets du groupe où les représentations se voient partagées et parfois confrontées.

\subsection{Des écrits au « terrain » et retour}

Des travaux plutôt récents sur le rôle des TIC en éducation aux problématiques socioenvironnementales ont été réalisés en géographie et en sciences de la vie. Les premiers s'intéressaient au rôle de la géomatique sur l'appréhension de notre environnement (Genevois, 2008), notamment à 
travers la notion d'hyperpaysage (Partoune, 2006), les seconds ont été orientés sur les pratiques de sciences participatives instrumentées pour une éducation scientifique (Legrand, 2013). Ces approches disciplinaires 4 ne se présentent pas de façon explicite dans leur fondement théorique5, comme des contributions au champ de recherche de l'éducation relative à l'environnement et/ou au développement durable (EEDD).

Or, même si nous savons qu'il existe pléthore recherches sur les TIC par ailleurs, nous croyons à l'intérêt de croiser explicitement les deux champs que sont les TIC et l'EEDD compte tenu des enjeux non seulement en termes éducatifs mais aussi en termes de rapport à la nature (Pruneau, Kerry, Freiman, Langis, et Cormier, 2016). Lorsque nous avons été missionnés pour former à l'intégration du numérique en EDD, nous avons donc effectué une recherche de la bibliographie6 centrée sur le croisement entre technologie et cette « éducations à », c'est-à-dire non disciplinaire 7 et non limitée aux milieux formels d'éducation. Elle nous a permis d'établir qu'en éducation relative à l'environnement et au développement durable francophone sensu stricto, les publications de recherche liées au rôle des TIC sont peu nombreuses. À ce croisement, différentes thématiques de recherche ont cependant émergé :

- le potentiel éducatif de la ludification par les « green games » (Genevois et Leininger-Frézal, 2010, 2013; Kramar, 2012; Godet, 2015) ;

-l'intérêt du travail collaboratif via des plateformes en ligne pour le développement des raisonnements socio-scientifiques complexes (Morin et Simonneaux, 2013) ou pour l'élaboration de cartes conceptuelles sur le développement durable (Ladage et Poplimont, 2015) ;

- le développement de compétences numériques pour une éducation à la citoyenneté mondiale (Genevois, 2013) ou pour allier l'éducation aux médias et à l'information et l'EDD (Kerneis, 2015) ;

- les potentialités des réseaux sociaux pour l'engagement socio-environnemental dans des contextes non scolaires (Pruneau, Et Jai, Khattabi, Benbrahim, et Langis, 2017; Touir, 2015).

Si toutes y contribuent, la plupart des publications recensées ne pose pas - comme le font par ailleurs des chercheurs sur les technologies éducatives lorsqu'ils débattent, par exemple, de l'impact des TIC sur la forme scolaire ${ }^{8}$ - la question fondamentale de la place des technologies informatisées en EDD : apportent-elles une plus-value pédagogique pour éduquer aux problématiques socioenvironnementales? Et si oui, par quelles propriétés? En 2016, un colloque intitulé «Vers de nouveaux modèles d'apprentissage, de pratiques pédagogiques innovantes et TIC pour l'EDD » (Ceci, Dumas, Touiaq, et Belahsen, 2017) semblait mettre au centre cette problématique mais seules deux contributions abordent explicitement les liens entre TIC et EDD : l'une sur les TIC comme outils pédagogiques montessoriens et dont les affordances seraient mobilisables pour développer un agir

\footnotetext{
${ }^{4}$ Le mot disciplinaire n'est ici pas réduit au sens de discipline scolaire.

${ }^{5}$ Sylvain Genevois (2008), par exemple, centre sa thèse sur les liens entre géomatique et géographie et se réfère ponctuellement à I'EDD comme un cadre possible, parmi d'autres, de l'application des SIG en classe.

${ }^{6}$ Établie à partir des portails Open edition Journals, Cairn et Google Scholar et par la combinaison des mots clefs « Technologies de l'information et de la communication » ou « numérique » et « Education au développement durable » ou « Education relative à l'environnement » ou « Education à l'environnement ».

${ }^{7}$ Cette méthode implique que les publications qui pourraient traiter de l'intégration des TIC dans les disciplines scolaires sur des sujets écosociaux (par exemple en SVT, les TIC et le changement climatique ou encore en technologie, les TIC pour l'établissement d'écobilan), sans référence explicite à l'EDD ou à l'ERE n'ont pas été retenues.

${ }^{8}$ Voir à ce propos le débat sur les TIC et les formes scolaire et universitaire dans la revue Distance et Médiation des savoirs (Peraya, 2018).
} 
favorable à l'environnement (Dumas, 2017) et l'autre analysant des sites internet potentiellement utilisables pour l'EEDD au Maroc (El Hafdi, Talbi, Radid, et Ihrachen, 2017).

Finalement, les résultats de notre exploration bibliographique me conduisent à affirmer que, pour le moment, la seule synthèse existante qui traite dans son ensemble la question de l'intégration des TIC avec une entrée EEDD est une publication de l'IFREE (Institut Formation Recherche Éducation à l'Environnement). Sous le titre « outils numériques et éducation à l'environnement » (Bauer et Girand, 2013), ce livret est construit sur les enquêtes de 10 expériences pédagogiques réalisées autant dans les milieux associatif que formel. Le propos ne prétend pas à l'exhaustivité mais interroge clairement: «Qu'est-ce que ces outils me permettent de faire de plus [en EEDD] que ce que je fais déjà avec des outils classiques »? (p. 6).

Enseignement à distance mis à part, un panorama des pratiques et des possibles dans le domaine est brossé, notamment à travers l'établissement d'une typologie des TIC, présentée plus bas. Les avantages en termes de motivation, de changement de posture des éducateurs, de possibilités pour le travail collaboratif sont entre autres abordés. Des points de vigilance sur le temps de préparation et sur l'équilibre à respecter entre l'usage des TIC et les objectifs éducatifs sont également émis, rejoignant ainsi d'autres analyses menées hors du champ de l'EDD (dont Tricot, 2017). La conclusion générale de l'IFREE sur l'impact des TIC en EDD est que « ce n'est pas l'outil lui-même qui est déterminant mais ce qu'on en fait » tout en affirmant que «l'outil » peut « sous-tend[re] déjà une intention pédagogique, et qu' [il] peut être porteur d'un modèle éducatif et de valeurs » (p. 127); laissant donc de fait le champ ouvert à de plus amples analyses.

Les apports théoriques de nos formations ont été bâtis à partir des travaux recensés et plus particulièrement à partir de la synthèse de l'IFREE. Nous y avons cependant largement insufflé nos cultures croisées en sciences de l'information et de la communication sur les médias informatisés, en sciences de l'ingénieur sur les objets communicants (Muracciole, 2009) ainsi que les analyses de nos pratiques auprès des scolaires (Zwang et Chamboredon, 2010). C'est à partir de ce matériau hybride qu'une réflexion sur les contenus et les pratiques de formation au «numérique » en EDD prend la forme, dans les lignes qui suivent, de recommandations. Il ne s'agit pas de (re)déployer ici tous les aspects de la formation des communautés éducatives dans ce domaine ${ }^{9}$ mais de focaliser sur ceux Éducation aux Médias et à l'Information (EMI) exceptée - qui concourent, selon nous, à un usage des TIC en EDD dans une perspective de transformation écosociale. Je présenterai donc dans ce cadre des propositions pour former de façon générale à l'usage des TIC en EDD puis je proposerai des pistes d'intégration des TIC pour favoriser le développement d'une écocitoyenneté critique.

\section{Propositions pour former à l'intégration des TIC en EDD}

\subsection{S'inscrire dans une approche culturelle et "pédago-centrée » des TIC}

\subsection{1. À propos du numérique en éducation : approche culturelle vs approche utilitariste}

Depuis les années 1970, les plans d'introduction des technologies en éducation se succèdent, marqués chacun par le vocable de leur époque : de «l'informatique » (1970) au «numérique » (2010) en passant par «l'internet» (1992) et les «TIC» (2000) (Plantard, 2016). Pour ne pas rester dans l'implicite d'un langage qui a fait passer très récemment l'adjectif « numérique », accommodé à l'envi, à un substantif masculin «le » numérique, une clarification du terme semble indispensable. Or il n'y a pas de définition consensuelle «du» numérique. Georges-Louis Baron (2017) le définit par ses composantes: les médias et l'information, l'informatique (la programmation et l'internet) et la

\footnotetext{
${ }^{9}$ Nous renvoyons notamment le lecteur à la proposition d'Yves Girault et al. sur la formation des enseignants en EDD, qui proposait plusieurs ancrages : épistémologiques, curriculaire, psychopédagogique et dans la didactique des questions socialement vives (Girault, Lange, Fortin-Debart, Delalande-Simonneaux, et Lebeaume, 2006)
} 
technologie (les objets connectés notamment) quand Milad Doueihi (Doueihi, 2013) fonde sa définition sur un nouvel humanisme, en insistant sur le partage des savoirs permis par une consubstantialité de l'industriel et du culturel. Avec de nombreuses divergences conceptuelles par rapport à cet auteur notamment dans son attachement à la dimension scripturale des communications, Yves Jeanneret (2011) considère les TIC comme des médias, avec une définition qui converge néanmoins vers cette dualité ontologique des médiations : " le média est un objet à la fois entièrement technique (car il est matériel et fabriqué par l'homme) et complètement social (car il conditionne l'échange social)» (p. 93). Enfin, Pascal Plantard (2016) pense que l'emploi de «numérique» (numerus : le nombre) peut traduire une vision du monde technocentrée quand la traduction anglaise «digital » (digitus : doigt) serait davantage anthropocentrée.

En formation, l'essence duale des TIC (techno $v s$ anthropocentré) auxquels les communautés éducatives ont affaire se traduit dans une dualité des approches : l'une, «techno-centrée », limitée à un objectif de maîtrise technique des instruments et médias numériques, l'autre, "pédago-centrée », qui prend en compte le contexte et les projets des enseignants, au regard d'objectifs pédagogiques (Charlier, 2010). À une échelle macroscopique et selon les catégories définies par Pierre Mœglin (2015), on pourrait dire que la première rend plus aisée une vision «productiviste » des activités éducatives instrumentées en se focalisant sur l'équipement comme moyen d'une optimisation de l'éducation, alors que la seconde s'ancre dans une vision «culturelle » de l'intégration des TIC, centrée sur les finalités éducative et in fine sur un projet de société humaniste.

Pour l'EDD, nous souhaitons privilégier cette dernière. C'est pourquoi l'intitulé de nos formations ne renvoie pas à des équipements ou des ressources comme par exemple «utiliser les tablettes » ou «utiliser la Banque de ressources numériques éducative $(\mathrm{BRNE})^{10}$ en $\mathrm{EDD}$ » mais à des visées pédagogiques: "Les outils collaboratifs numériques en EDD : diversité et potentialités pédagogiques » ou encore «Le numérique comme appui aux débats entre les élèves ». Ils marquent, de fait, une distance par rapport à la promotion d'équipements financés en amont, comme peuvent l'être les flottes de tablettes du dernier plan numérique pour l'École (Plantard, 2016). Ce faisant, nous tentons «d'instrumenter l'enseignement sans l'instrumentaliser» (Mœglin, 2015, p. 70) au profit de logiques de déploiement, dans les murs de l'École, de marchés et de services de la culture qui pourraient essentiellement servir aux industries éducatives (éditeur scolaire, fabriquant de matériels informatiques, éditeurs de logiciels non libres, etc.) (Mœglin, 2015). Cela implique également de prendre de la distance face à des discours positivistes qui feraient des TIC la clef de voute de l'avènement d'un développement durable comme expression d'une croissance économique permise par une plus grande efficacité de l'éducation (Berhault, 2011). Et cela, en questionnant le projet éducatif sous-tendu, soit l'EDD elle-même.

\subsection{2. À propos de l'EDD : clarifier le projet éducatif avant de «l'instrumenter »}

Avant de lier EDD et numérique, l'introduction de notre première formation avait pour objet d'interroger cette éducation en milieu formel. En effet, le changement de paradigme institutionnel de l'éducation à l'environnement vers l'éducation au développement durable a suscité des interrogations voire des oppositions autant dans les milieux associatifs qu'académiques. Elles portaient non seulement sur l'objet théorique développement durable (Sauvé, 2008; Alpe, 2011) mais aussi sur les savoirs associés, souvent « ressourcistes » (Girault, 2012), et les pratiques éducatives très pragmatiques comme moyens de combler la vacuité épistémique de l'objet théorique (Barthes, Zwang, et Alpe, 2013).

Initier une réflexion sur le cadre prescriptif de l'EDD en milieu formel avant d'intégrer des objets techniques nous paraît essentiel. Alors que différents positionnements épistémologiques (Girault et Sauvé, 2008), éthiques et d'objectifs (Girault, Zwang, et Jeziorski, 2013) peuvent exister en EEDD,

${ }^{10}$ Banque de ressources numériques éducative issue d’un partenariat entre des éditeurs privés et l'Éducation nationale. 
quelles finalités, valeurs, savoirs et approches pédagogiques sont promus institutionnellement en EDD ? Dans quelle histoire s'inscrit-elle ? (Girault et Fortin-Debart, 2006; Barroca-Paccard, 2013). Comment, en tant que théorie éducative pensée par l'Éducation nationale, a-t-elle évoluée depuis la première circulaire de généralisation? Clarifier les attendus institutionnels dans leurs dimensions téléologique, axiologique, praxéologique et épistémologique (Zwang et Girault, 2012) ainsi que leurs évolutions, même de manière brève, c'est concourir à un positionnement des acteurs éducatifs en amont de l'instrumentation, ce qui situe d'emblée l'action de formation dans un cadre de finalités éducatives et de valeurs explicitées. Nous optons donc pour une démarche pluraliste, d'analyse pédagogique et critique de l'utilisation des TIC, comme une des modalités possibles pour cette éducation.

\subsection{Identifier la diversité des TIC : entre pédagogie et industrie}

Pour privilégier un ancrage pédagogique des formations, il semble pertinent que les enseignants puissent se repérer parmi les TIC dont ils disposent. Pour cela, comme je l'ai évoqué plus haut, une typologie basée sur les usages a été proposée par l'IFREE (Bauer et Girand, 2013). Elle catégorise les TIC en « outils » :

- de lecture, qui permettent la consultation d'un contenu (texte, image, audio, vidéo) ;

- de recherche d'informations dans une pluralité (moteurs de recherche);

- d'écriture, comprenant la collecte, la production et la publication d'informations en créant des fichiers (images, vidéos, sons), en les organisant puis en les communiquant en ligne ;

-d'échange, basés sur la communication synchrone ou asynchrone d'informations (mails, forums, réseaux sociaux) ;

- de compréhension, qui proposent des modélisations de la réalité (expérimentation assistée par ordinateur) ;

- d'apprentissage à partir de consignes, ce que permettraient les exerciseurs, les tutoriels et les jeux sérieux.

Si les quatre premières catégories renvoient plutôt à des fonctions communicationnelles, les deux dernières s'ancrent dans l'activité cognitive de l'élève, selon deux présupposés : les modélisations faciliteraient la compréhension et les exerciseurs, tutoriels et autres jeux sérieux généreraient un apprentissage. Or pour ne s'attarder que sur les jeux sérieux, le lien entre activité ludique et apprentissage, s'il est recherché (Alvarez, 2014), y compris en EDD à travers les «green games » (Genevois et Leininger-Frézal, 2013), ne fait pourtant pas l'unanimité dans les études menées (Lavigne, 2016; Schmitt, 2016). Par ailleurs, Erica de Vries (2001), en classant les logiciels d'apprentissage selon huit fonctions pédagogiques ${ }^{11}$, classe les ludiciels dans la catégorie des objets capables en eux-mêmes de captiver l'attention et de susciter une motivation plutôt qu'un apprentissage, ce que confirment les récents travaux de Damien Djaouti (Djaouti, 2016). Sur la base de cette analyse bibliographique, nous avons donc élaboré une typologie provisoire, inspirée de celle de l'IFREE ${ }^{12}$. On voit bien ici comment le caractère hybride du GIPTIC EDD a été mis à profit pour tenter d'élaborer une classification opératoire des « outils » tout en prenant en compte une approche critique de leurs usages.

\footnotetext{
${ }^{11}$ Soit : présenter de l'information; dispenser des exercices répétés ; enseigner par des tutoriels intelligents ; captiver l'attention et la motivation de l'élève par des jeux ; fournir un espace d'exploration par des hypermédias ; fournir un environnement pour la découverte de lois naturelles par des simulations ; fournir un environnement pour la découverte de domaines abstraits par la construction d'objets; et enfin fournir un espace d'échange entre élèves.
}

\footnotetext{
12 À noter que les « outils de lecture » ne sont pas différenciés car ils sont implicites dans l'accès qu'ils permettent aux supports médiatiques).
} 
Ainsi, en formation initiale, un atelier d'initiation aux TIC en EDD a été conçu autour d'une grille (Tableau 1) permettant : le repérage d' « outils », la conception sommaire d'une activité pour chacune des catégories, l'explicitation de ses objectifs pédagogiques et l'analyse a priori des gains et des limites de leur utilisation dans le cadre choisi. Parmi les usages présentés, une lacune est à noter : quid de l'évaluation? Il n'est évidemment pas l'objet de discuter plus avant ici de cette typologie mais ces remarques justifient les modifications que nous lui avons apportées.

\begin{tabular}{|c|c|c|c|c|c|}
\hline Fonction & Permettent de & Exemple & $\begin{array}{l}\text { Activité } \\
\text { proposée }\end{array}$ & $\begin{array}{l}\text { Objectif(s) } \\
\text { pédagogi- } \\
\text { que(s) }\end{array}$ & $\begin{array}{c}\text { Gain(s) et/ou } \\
\text { limite(s) }\end{array}$ \\
\hline $\begin{array}{l}\text { Collecte de } \\
\text { données }\end{array}$ & Capter et enregistrer des données & & & & \\
\hline Écriture & $\begin{array}{l}\text { Produire, gérer et organiser des } \\
\text { contenus }\end{array}$ & & & & \\
\hline Simulation & $\begin{array}{l}\text { Simuler des phénomènes à partir } \\
\text { de modélisations }\end{array}$ & & & & \\
\hline $\begin{array}{c}\text { Recherche } \\
\text { d'informations }\end{array}$ & $\begin{array}{l}\text { Rechercher parmi une multitude } \\
\text { de contenus disponibles }\end{array}$ & & & & \\
\hline Échange & $\begin{array}{l}\text { Lire, écrire et réagir de façon } \\
\text { synchrone ou asynchrone }\end{array}$ & & & & \\
\hline $\begin{array}{l}\text { Enseignement } \\
\text { (didacticiel) }\end{array}$ & $\begin{array}{l}\text { Suivre des consignes, avec ou } \\
\text { sans interaction, dans le but } \\
\text { d'acquérir des connaissances, des } \\
\text { capacités voire des attitudes }\end{array}$ & & & & \\
\hline $\begin{array}{l}\text { Ludique } \\
\text { (ludiciel) }\end{array}$ & $\begin{array}{l}\text { Jouer dans le cadre d'un scénario } \\
\text { utilitaire dans le but d'acquérir } \\
\text { des connaissances, des capacités } \\
\text { voire des attitudes }\end{array}$ & & & & \\
\hline Évaluation & $\begin{array}{l}\text { Évaluer les connaissances et les } \\
\text { capacités mobilisées }\end{array}$ & & & & \\
\hline
\end{tabular}

Tableau 1. Exemple de grille d'analyse des TIC en EDD

De plus, présenter une liste «d'outils» ne suffit évidemment pas. Ils doivent être considérés dans les possibilités et les limites de leur contextualisation pédagogique, ce que le livret de l'IFREE questionne comme étant leur possible plus-value. En effet, dans quelles conditions est-il pertinent de concevoir une activité avec des TIC plutôt que sans ? Dans quelle mesure y a-t-il cohérence entre le dispositif choisi et les objectifs pédagogiques visés ? Quels sont les obstacles liés à son intégration dans la séquence pédagogique, notamment en termes temporels ou techniques ? Comment les contourner, notamment avec les retours d'expériences et les idées des autres participants ? Autant de questions qui, même sans réponses immédiates, méritent d'être soulevées.

À l'occasion de cette analyse, il est pertinent d'attirer l'attention sur les modèles socio-économiques des producteurs des dispositifs éducatifs choisis : paiement à l'unité, abonnement, financement par la publicité ou gratuité (Mœglin, 2004), et de les mettre en regard avec leurs finalités. L'étude de la circulation des expositions itinérantes en milieu scolaire a par exemple permis de montrer que derrière la gratuité se jouaient des légitimités dont les ressorts sont parfois très éloignés des principes éducatifs de 1'EDD institutionnelle (Zwang, 2016). Des transpositions de ces analyses sont possibles pour les TIC, comme par exemple pour les jeux sérieux développés par Deyrolle avec le soutien de l'Éducation nationale (Figure 1), comme fruit d'une alliance construite autour du rayonnement d'un éditeur dans 
l'objectif, contraire aux principes de l'approche systémique promue par l'éducation nationale, d'inculquer des gestes. Mais avec les dispositifs numériques, la problématique de la gratuité prend d'autres dimensions, entre les projets développés sous licences libres et les possibilités de collecte des données laissées par les utilisateurs aux fins privées de certains éditeurs.

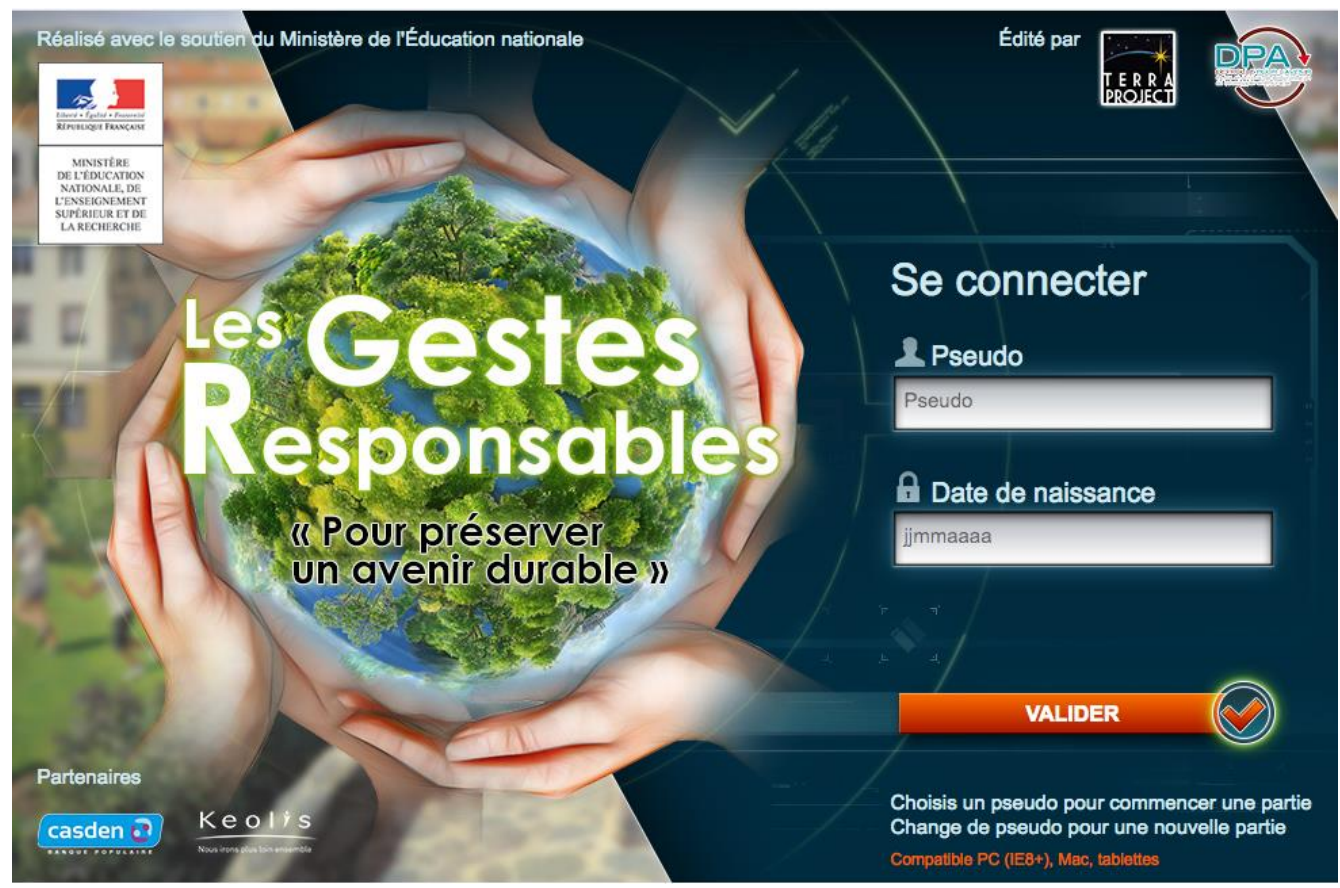

Figure 1. Page d'accueil d'un " green game » réalisé par Deyrolle et légitimé par l'Éducation nationale

Initier aux enjeux de l'industrialisation de l'éducation, largement méconnus du monde éducatif et des enseignants en particulier (Mœglin, 2016), c'est éveiller à une approche non utilitariste des TIC qui ne reposerait donc pas sur l'essor à tout crin des industries éducatives, tant ce projet semble difficilement compatible avec l'avènement d'une écocitoyenneté critique. C'est s'inscrire dans la cohérence d'une approche culturelle permettant «de transmettre, partager, entretenir et enrichir les valeurs de la civilisation et les normes du vivre ensemble $»$ (Mœglin, 2015, p. 70). La prochaine partie permettra de présenter des éléments sur des dispositifs propices au développement de compétences critiques, éthiques et politiques.

\section{Propositions pour former à une intégration des TIC favorisant le développement d'une écocitoyenneté critique}

\subsection{Former aux TIC permettant la coopération et la collaboration}

Selon Lucie Sauvé, «l'un des apprentissages majeurs de l'écocitoyenneté est [...] celui de la valorisation du collectif dans l'apprentissage et l'action » (Sauvé, 2013, p. 33). Certains instruments et médias numériques sont aptes à soutenir cette dynamique collégiale : ceux d'échanges (Tableau 1), qui permettent de partager des idées en vue d'une réflexion collective, ceux de collecte de données, qui dans leurs versions communicantes, rendent possibles les mutualisations, et ceux d'écriture, qui, orientés vers une production commune, offrent divers degrés de travail en équipe : de la coopération, par l'adjonction des apports identifiés de chacun des participants, à la collaboration, derrière laquelle les contributions individuelles se fondent dans la réalisation conjointe (Connac, 2017).

Orienter les formations vers les potentialités collaboratives permises par les activités intégrant des logiciels d'écriture semble donc judicieux. Pour nous, il s'agit autant de former aux logiciels de mindmapping, de cartographie participative, d'écriture en ligne type wiki, «drive », « online » ou murs collaboratifs comme supports de communication éducative que de présenter les fonctionnalités 
permises par les gestionnaires de tâches (type Trello13) pour les inscrire et les répartir entre les membres de projets de classe ou d'établissement. L'utilisation de ces derniers en éducation nécessite de prévoir que les élèves s'assignent eux-mêmes les tâches ou après discussion avec les membres de la communauté éducative, au risque que ces soutiens à une forme d'ingénierie n'engendrent une organisation trop rationnalisée et descendante.

En seconde, dans un groupe de MPS (Méthodes et pratiques scientifiques), nous avons eu l'usage de différentes TIC en lien avec la visite d'une mare urbaine. En amont, nous avons élaboré collectivement une carte heuristique numérique, puis la visite a été instrumenté par des appareils de mesure (oxymètre, thermomètre électronique) et les téléphones portables des élèves (pour garder des traces de la visite par des photographies), ce qui a permis l'élaboration d'un compte rendu synchrone sur un «mur » (Figure 2). Les TIC ont été des appuis à la constitution d'un collectif de travail. L'analyse de cette séquence semble montrer que la mise en média par les TIC, comme visée (prendre des photographies pour préparer le compte rendu) et comme production (le compte rendu numérique lui-même), permettrait une objectivation des coopérations apte à en soutenir la prise de conscience et l'épanouissement. En ce sens, l'utilisation combinée des TIC pour la documentation de traces communes permettrait une forme d'acculturation à la pensée collégiale (Zwang, 2019).

${ }^{13}$ https://trello.com/ 


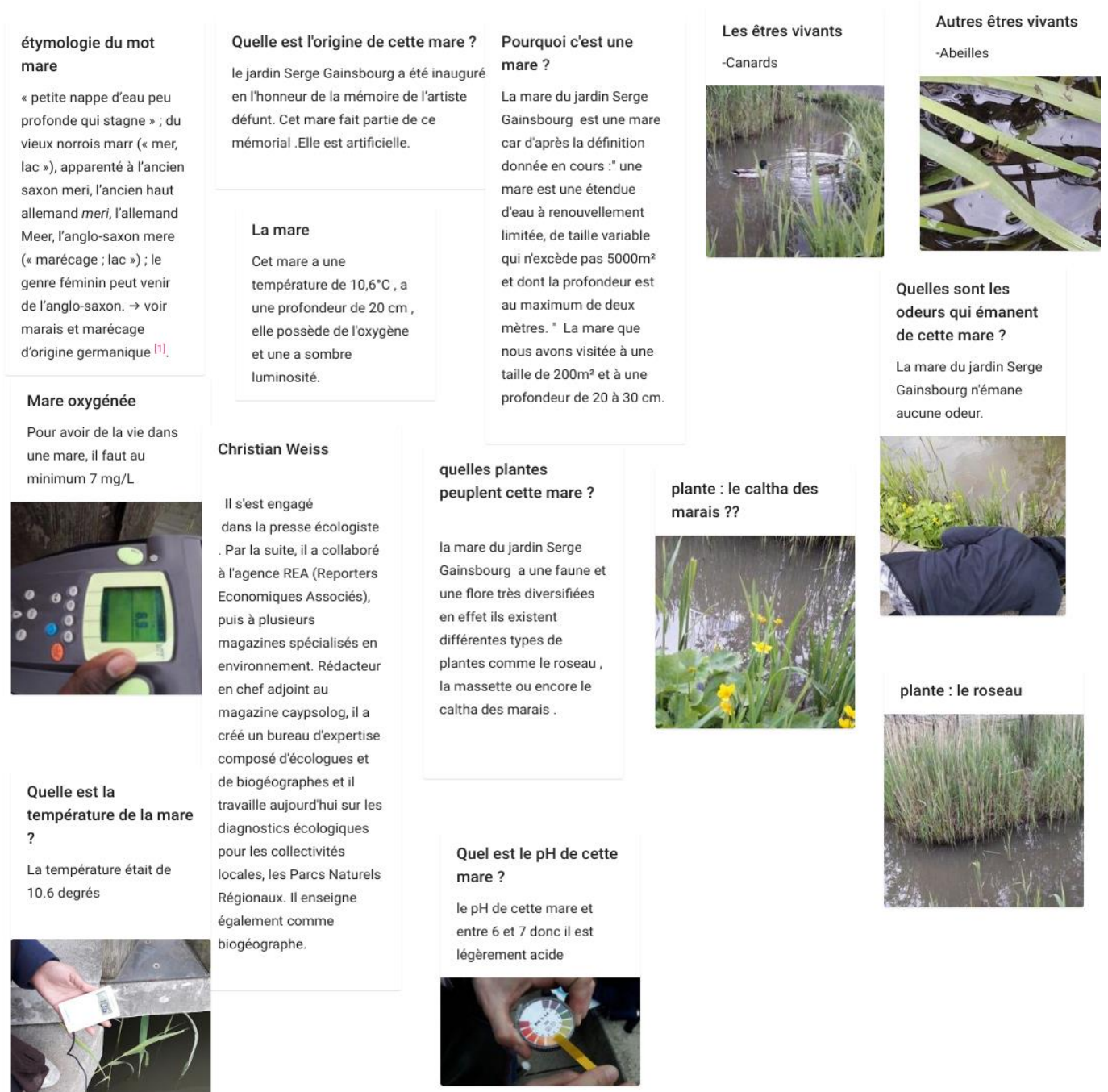

Figure 2. "Mur » réalisé par les élèves du groupe de MPS suite à la visite de la mare du jardin Serge Gainsbourg (Visible ici : https://fr.padlet.com/aurelie_zwang/kfr8dwkqrOma. Consulté le 15 décembre 2018)

\subsection{Former auX TIC soutenant la mise en débat et la prise de décision collective}

Si les compétences critique et politique n'ont pas besoin du numérique pour être exercées en classe, certaines TIC sont en mesure de renforcer des dispositifs éducatifs élaborés autour du débat et de la prise de décision collective, soit car elles ont été spécifiquement conçues dans cette optique, soit car ce type d'activité peut reposer sur les propriétés communicationnelles d'instruments numériques plus généralistes. Dans une perspective de formation au numérique à visée sociocritique, cela implique qu'il faille non seulement initier aux uns mais aussi développer et partager des contextualisations pédagogiques pour les autres. 
À titre d'exemple parmi les TIC développées pour s'intégrer dans une séquence pédagogique reposant sur la confrontation d'arguments, citons le logiciel C-Roads. Ce logiciel de simulation sert d'appui à un scénario de négociations fictives sur le changement climatique. En France, il a été surtout utilisé au moment de la COP 21, notamment lors d'un projet inter-académique francilien (Thizeau, 2015), lorsque les instructions officielles prescrivaient des jeux de rôle en classe pour publiciser l'accord de Paris (MENESR - DGESCO, 2015). En entrant les objectifs chiffrés de réduction des émissions de gaz à effet de serre (en équivalent $\mathrm{CO} 2$ ) issues des politiques publiques aux échelles nationale et régionale (Union européenne par exemple), le programme permet d'en observer instantanément l'impact sur la température moyenne de la Terre à l'horizon 2100 (Figure 3).

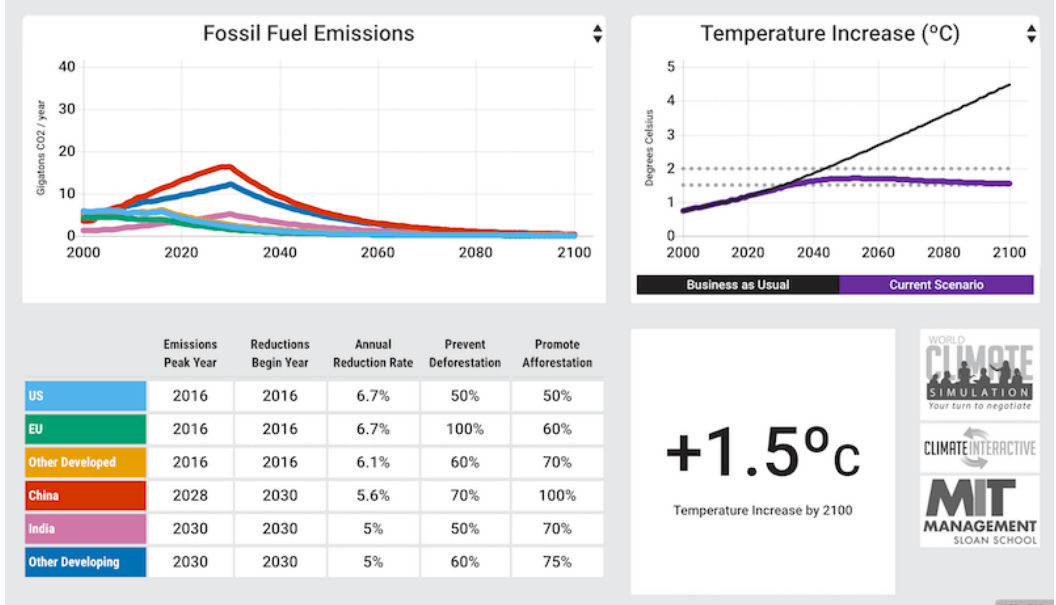

Figure 3. Capture d'écran d'une simulation réalisée par le logiciel C-Roads (source : https://www. climateinteractive.org/tools/c-roads/)

Du côté des TIC plus généralistes, évoquons l'expérimentation que nous avons réalisée en employant Plickers ${ }^{14}$, un outil dont la fonction principale est l'évaluation par QCM. Il ne s'agit pas de relater ici toute la démarche ${ }^{15}$ mais de montrer l'intérêt d'avoir rendu des élèves de $3^{\mathrm{e}}$ acteurs, et non pas uniquement spectateurs, d'un débat sur l'énergie entre des élèves de $2^{\text {nd }}$. Ces derniers devaient présenter les orientations énergétiques d'un parti politique imaginaire pour répondre aux défis énergétiques actuels et futurs devant les élèves d'une classe de $3^{\mathrm{e}}$. Ces propositions étaient en réalité issues de choix politiques réels, émis en Allemagne, aux États-Unis et en France (dans les programmes de candidats aux primaires en vue de l'élection présidentielle de 2017), ce que les élèves de seconde ont ignoré jusqu'à un dévoilement après le débat.

Les élèves de $3^{\mathrm{e}}$ ont donné leur avis en temps réel sur la qualité des arguments avancés et sur l'expression orale de chacun des partis joués, grâce à des QR code scannés par un Smartphone. Puis, de la même façon, ils ont voté pour un parti : contre toute attente, celui qui présentait les options les moins compatibles avec une transition énergétique, soit une sorte de «business as usual», a été élu. Sans juger à aucun moment leur choix, la comparaison entre les appréciations des élèves de $3^{\mathrm{e}}$ sur chaque parti et ce résultat a permis de mettre immédiatement en évidence que le principal critère de choix ne reposait pas sur le contenu des arguments mais sur leur énonciation par des débatteurs convaincus, ce qui, de notre point de vue, a constitué un révélateur très important capable de contribuer à leur éducation au politique.

En formation, il semble donc opportun de faire élaborer des scénarios pédagogiques construits autour de la médiatisation synchrone d'une activité collective de négociation ou de débat comme

\footnotetext{
${ }^{14}$ https://www.plickers.com/

${ }^{15}$ Un article et une vidéo en résument les principales étapes : https://www.ac-paris.fr/portail/jcms/p1 1631233/appuyer-lesenjeux-pedagogiques-du-debat-de-classe-avec-des-outils-numeriques. Consulté le 11 février 2018.
} 
catalyseur d'une réflexion critique avec et entre les élèves. De cette condition résulte que la médiation numérique par les TIC s'incorpore comme plus-value pédagogique à l'exercice d'une «disputatio » et non comme un apparat technologique (Zwang, 2019).

\subsection{Former aux communs (par le) numérique(s)}

Dans la perspective de développer une éthique du commun, nous partageons l'idée de Louise Merzeau et d'Hélène Mulot selon laquelle «l'institution scolaire doit se départir d'une conception instrumentale du numérique [...] pour le repenser comme un environnement à investir et transformer collectivement» (2017, p. 78). Dans la continuité des pratiques de classe favorisant les travaux collaboratifs, une sensibilisation des élèves aux problématiques culturelle et politique de la production et de la circulation des savoirs devrait donc nécessairement s'accompagner aussi d'une formation des communautés éducatives aux communs numériques et par le numérique; la notion de communs pouvant s'étendre à l'ensemble des biens communs (eau, air, services publics) ce qui en constitue non seulement l'intérêt mais aussi l'enjeu.

Une première voie - implicite - de formation aux communs tente de créer les conditions de possibilité de la co-formation, en présentiel et à distance, à travers des espaces d'échanges en ligne ayant pour fonction de recueillir les points de vue, les connaissances et les expériences d'encadrants de projets d'EDD. Avec comme objectif de contribuer à créer une dynamique basée sur des «capacités latérales » («we $[. .$.$] describe lateral capacity across schools, were principals and teachers leaders$ collaborate with other schools to learn from and to contribute to school improvement, not only in individual schools, but also in the district as a whole. » (Fullan, 2005, p. 17)). Nous avons obtenu de l'académie la création d'un espace numérique réservé aux référents des écoles et des établissements scolaires, que nous avons structuré en trois grandes parties (document 4). Cet espace n'est pas à proprement parler inclus dans un dispositif hybride mais il permet d'ouvrir ou de prolonger la réflexion sur des sujets abordés en présentiel. Il s'agit de concourir à l'intégration de mutualisations via le numérique dans les pratiques professionnelles pour que diffuse ce type de modalités dans les pratiques de classe. N'étant pas courante, elle se confrontent nécessairement à des limites, dans la participation notamment (Baron et Bruillard, 2004).

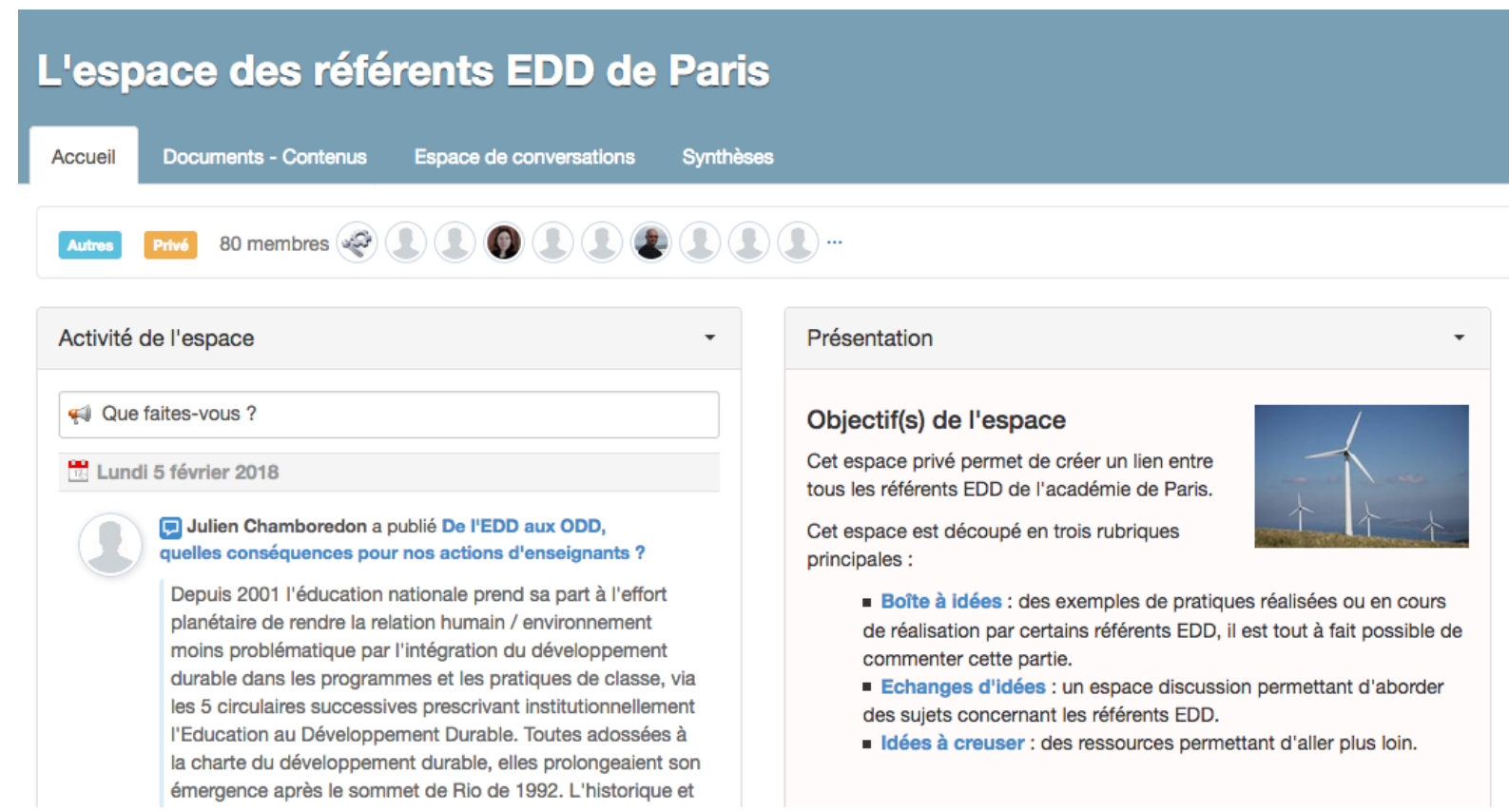

Figure 4. Capture écran de l'espace des référents EDD de l'académie de Paris présentant les trois grandes rubriques ainsi qu'une discussion en cours.

Une deuxième voie - explicite - de formation aux communs consiste à acculturer aux logiciels libres. Sans entrer dans les détails de programmation, il s'agit d'en expliquer la philosophie, soit les 
libertés logicielles d'exécuter, d'étudier, de redistribuer et de modifier des codes sources à des fins d'amélioration pour en faire bénéficier une communauté d'utilisateurs, en partant du principe que la création et la connaissance sont des biens culturels communs. Cela s'inscrit dans une volonté de promouvoir des alternatives aux licences propriétaires déjà fort implantées dans le service public d'éducation tout en faisant prendre conscience de l'enjeu politique du contrôle des logiciels et des données par les citoyens. Les différentes licences permettant d'utiliser et de produire des ressources éducatives, comme celles de l'association Creative Commons (Boyer, 2017) peuvent être à cette occasion explicitées. Pour une même visée pédagogique, en formation, il semble également équitable de présenter systématiquement des TIC équivalents sous les deux types de licence, privatives et libres, nonobstant quelques pertes de fonctionnalités que les seconds ont parfois par rapport aux premiers, ce qui limite de fait leur diffusion.

\section{En guise de conclusion : de la nécessaire formation aux limites du numérique pour une EDD à visée écosociale}

Cet article avait pour objectif de dégager des points saillants de la formation au numérique en ÉDD des communautés éducatives. Il semble que les TIC, employées avec pertinence, puissent en effet être un ferment d'une transformation écosociale, via le développement de l'esprit critique des élèves, de leur coopération et d'une acculturation aux communs, comme autant de compétences sur lesquelles pourrait s'adosser un engagement en faveur des problématiques socio-environnementales. Cela justifie une formation spécifique au sein d'une École. Avec cet objectif, Le GIPTIC EDD souhaite créer un réseau d'établissements mesurant la qualité de l'air extérieure à partir d'objets communicants élaborés sur des cartes électroniques Open source. S'adressant à tout enseignant souhaitant y inscrire son établissement, l'accompagnement serait modulaire : du soutien à l'exploitation pédagogique des mesures à l'aide à la démarche technologique de fabrication des objets par les élèves, en passant par l'assistance à la mise en réseau entre les établissements participants. S'il aboutit tel que nous le concevons actuellement, ce projet s'inscrirait dans le développement d'une éco-citoyenneté critique à triple titre :

- les élèves généreraient les données, ce qui les mettrait en posture de produire eux-mêmes un savoir environnemental ;

- ce savoir serait partagé entre établissements, ce qui contribuerait à développer un sentiment d'appartenance à un collectif ;

- les élèves seraient initiés à un internet des objets conçu à partir d'un code et d'un matériel libres, à l'heure où les progrès de la domotique interrogent sur les possibilités d'intrusion dans les vies privées.

Notre objectif est de faire de l'échange de données produites par les élèves entre les établissements scolaires un enjeu de transformation écosociale, dans ce qu'elles induisent comme questionnement sur le type de technologies employées et comme valeur symbolique dans la mesure où le réseau, dénommé «Scol'air», engendrerait comme pouvoir de comparaison et de discussion avec les valeurs communiquées par l'organisme chargé de la surveillance de la qualité de l'air. Sans a priori aucun sur la fiabilité de cette structure, de récentes études - le rapport Fos Epseal (Allen, Cohen, Ferrier, et Lees, 2017) ou encore le livre de Chantal Pouliot (2015) - ont en effet montré que des mesures réalisées par les citoyens eux-mêmes ont pu révéler des valeurs de polluants que les opérateurs avaient occultées, ce qui constitue une base objective à partir de laquelle des actions collectives peuvent se structurer.

Malgré l'intérêt possible de cette intégration des TIC, il convient de s'interroger: dans quelle mesure cette intégration des TIC en EDD ne constitue pas en elle-même un obstacle à la transformation souhaitée ? En effet, l'appréhension des situations socio-environnementales par le truchement d'objets techniques prend constamment le risque de la promotion de pratiques pédagogiques où la médiation technologique entre les humains et la nature serait érigée en modus vivendi. Or dans quelle mesure la systématisation de cette relation indirecte ne peut-elle pas faire écran au développement de rapports d'attachement à la nature et donc à sa protection ? Dans un contexte où les enseignants semblent eux- 
mêmes moins en contact avec les milieux physiques qu'avec les environnements numériques (Pruneau et al., 2016), cette préoccupation s'étendrait même aux éducateurs. De plus, n'est-il pas éminemment paradoxal de penser contribuer à une transition socio-écologique par les TIC lorsque les émissions de gaz à effet de serre, les pollutions extractives et les déchets qu'elles génèrent entretiennent des inégalités Nord-Sud autant que les dégradations environnementales globales ? Finalement, une formation parachevée du numérique en EDD ne pourrait-elle pas aussi être une formation à ses alternatives ? Ce programme est certes ambitieux mais à la hauteur des enjeux d'une transition socioécologique éclairée.

\section{Bibliographie}

Allen, B., Cohen, A. K., Ferrier, Y., et Lees, J. (2017). FOS EPSEAL. Étude de participation en santé environnementale ancrée localement sur le front industriel de Fos-sur-Mer et Port-Saint-Louis-du-Rhône. Centre Norbert Elias, UMR 8562. Consulté à l'adresse https://f.hypotheses.org/wp-content/blogs.dir/3282/files/2017/01/FOS-EPSEAL-ANSES16-1-2017-logo-red.pdf

Alpe, Y. (2011). Le « curriculum sournois » du développement durable. Dans B. Bader et L. Sauvé (dir.), Éducation, environnement et développement durable : vers une écocitoyenneté critique (p. 103-122). Québec, Canada : Presses de l’Université Laval.

Alvarez, J. (2014). Serious Game: questions et réflexions autour de son appropriation dans un contexte d'enseignement. Psychologie Clinique, (37), 112-126.

Baron, G.-L, et Bruillard, E. (2004). Quels apprentissages dans des communautés d'enseignants en ligne ? Réflexions méthodologiques et perspectives. Dans B. Charlier et A. Daele, Comprendre les communautés virtuelles d'enseignants : Pratiques et recherches (p. 177-197). France, Paris : L'Harmattan.

Baron, G.-L. (2017). Former et accompagner des enseignants au numérique : une entreprise vouée à l'échec ? Présenté à Numérique et nouveaux enjeux de la formation, journée d'étude de l'ESPÉ.

Barroca-Paccard, M. (2013). Les programmes scolaires: de l'Éducation à l'environnement vers l'éducation au développement durable (1970 - aujourd'hui). Dans C.-F. Mathis et J.-F. Mouhot (dir.), Une protection de la nature et de l'environnement à la française ? (XIXe-XXe siècles) (p. 281-291). Seyssel : Champ-Valon.

Barthes, A., Zwang, A., et Alpe, Y. (2013). Sous la bannière développement durable, quels rapports aux savoirs scientifiques ? Éducation Relative à l'Environnement : regards, recherches, réflexions, 11, 81-98.

Bauer, A., et Girand, R. (2013). Outils numériques et éducation à l'environnement: Quels usages possibles avec le public? Institut de Formation et de Recherche en Éducation à l'Environnement en Poitou-Charentes. Consulté à l'adresse http://www.ifree.asso.fr/images/publications/livrets/pdf/livret-ifree-n5.pdf

Berhault, G. (2011). Développement durable 2.0: L'internet peut-il sauver la planète? La Tour d'Aigues : Éditions de l'Aube.

Boyer, A. (2017). Une brève histoire des ressources éducatives libres. Administration et Éducation, (146), 119-124.

Bruillard, É. (2012). Lire, écrire, computer : émanciper les humains et contrôler les machines. E dossier de l'audiovisuel : l'éducation aux cultures de l'information. Consulté à l'adresse https://edutice.archivesouvertes.fr/file/index/docid/826643/filename/a1209d.htm

Ceci, J.-F., Dumas, P., Touiaq, M., et Belahsen, Y. (2017). Vers de nouveaux modèles d'apprentissage, de pratiques pédagogiques innovantes et TIC pour l'éducation au développement durable. Consulté à l'adresse https://archivesic.ccsd.cnrs.fr/sic_01660505/document

Charlier, B. (2010). Les TIC ont-elles transformé l'enseignement et la formation ? Dans F. Henri et B. Charlier (dir.), Apprendre avec les technologies (p. 145-156). Paris : Presses Universitaires de France.

Connac, S. (2017). Apprendre avec les pédagogies coopératives : Démarches et outils pour l'école. ESF Sciences humaines.

Crozier, M., et Friedberg, E. (2014). L’Acteur et le système. Les Contraintes de l'action collective. Paris : Points.

De Vries, E. (2001). Les logiciels d'apprentissage : panoplie ou éventail ? Revue française de pédagogie, (137), 105-116.

Djaouti, D. (2016). Serious Games pour l'éducation : utiliser, créer, faire créer ? Tréma, (44), 51-64.

Doueihi, M. (2013). Qu'est-ce que le numérique? Paris : Presses Universitaires de France. 
Fourgous, J.-M. (2012). « Apprendre autrement » à l'ère numérique. Se former, collaborer, innover : un nouveau modèle éducatif pour une égalité des chances. Paris.

Fullan, M. (2005). Leadership et Sustainability: System Thinkers in Action. Corwin Press.

Genevois, S. (2008). Quand la géomatique rentre en classe. Usages cartographiques et nouvelle éducation géographique dans l'enseignement secondaire. Thèse de doctorat. Université Jean Monnet - Saint-Etienne.

Genevois, S. (2013). Culture numérique et citoyenneté mondiale : quels enjeux pour l'École ? Tréma, (40), 16-27.

Genevois, S., et Leininger-Frézal, C. (2010). Les «serious games » : un outil d'éducation au développement durable ? Présenté à Colloque International «Éducation au développement durable et à la biodiversité : concepts, questions vives, outils et pratiques », Digne les Bains.

Genevois, S., et Leininger-Frézal, C. (2013). Introduction des «jeux sérieux » à l'école: vers un nouveau rapport au savoir? Présenté à Actualité de la Recherche en Éducation et Formation, Montpellier.

Gilbert, A.-F. (2018). Le travail collectif enseignant, entre informel et institué. Dossier de veille de l'IFÉ, 124, 40.

Girault, Y. (2012). Environnement et développement: présupposés épistémologiques et retombées éducatives des Travaux d'Initiative Personnelle Encadrés. Dans B. Bader et L. Sauvé (dir.), Éducation, Environnement et Développement Durable : vers une écocitoyenneté critique (Les Presses de l'Université Laval, p. 313-342). Laval.

Girault, Y. (2017). La recherche contemporaine en éducation relative à l'environnement au sein de la Francophonie : Où en est-on? Présenté à 6e Forum Planet'ERE, Montréal.

Girault, Y., et Fortin-Debart, C. (2006). État des lieux et perspectives en matière d'Éducation Relative à l'Environnement à l'échelle nationale. Consulté à l'adresse http://www.yvesgirault.com/pages/doc-pdf/rapport ere edd.pdf

Girault, Y., Lange, J.-M., Fortin-Debart, C., Delalande-Simonneaux, L., et Lebeaume, J. (2006). La formation des enseignants dans le cadre de l'éducation à l'environnement pour un développement durable : problèmes didactiques. Education Relative à l'Environnement : regards, recherches, réflexions, (6), 119-196.

Girault, Y., et Sauvé, L. (2008). L'éducation scientifique, l'éducation à l'environnement et l'éducation pour le développement durable : Croisements, enjeux et mouvances. Dans Y. Girault et L. Sauvé (Dir.), Aster - L'éducation à l'environnement ou l'éducation au développement durable (vol. 46, p. 7-30). Lyon : INRP.

Girault, Y., Zwang, A., et Jeziorski, A. (2013). Finalités et valeurs de différentes politiques d'éducation à la soutenabilité. Éducation Relative à l'Environnement : regards, recherches, réflexions, 11, 61-80.

Godet, F. (2015). Etude du potentiel des Serious Games pour la sensibilisation au développement durable. Mémoire UCL. Consulté à l'adresse https://dial.uclouvain.be/memoire/ucl/en/object/thesis\%3A2615/datastreams

Jeanneret, Y. (2011). Y-a-t-il (vraiment) des technologies de l'information? Nouvelle édition revue et corrigée. Presses Universitaires du Septentrion.

Kerneis, J. (2015). Le «savoir-devenir» un pont entre EMI et EDD dans le nouveau design de l'école de 2015 ? Présenté à Colloque international francophone «former au monde de demain. Quelles compétences, communes ou spécifiques, entre l'éducation et la formation au développement durable et les autres éducations et formations à la solidarité, la santé, les médias et la gouvernance ». Consulté à l'adresse https://halshs.archives-ouvertes.fr/halshs$\underline{01336828 / \text { document }}$

Kramar, N. (2012). Apport d'un jeu sérieux pour l'instauration d'un nouveau rapport au savoir du point de vue épistémologique : cas du jeu Clim@ction. Master 2 Histoire, Philosophie, Didactique des Sciences. Lyon I.

Ladage, C., et Poplimont, C. (2015). Le travail collaboratif sur des cartes conceptuelles en libre accès sur le Web comme réponse à la complexité de l'éducation au développement durable. Dans Enjeux et usages des technologies de l'information et de la communication (EUTIC). Fort de France, France : Serge Agostinelli. Consulté à l'adresse https://hal-amu.archives-ouvertes.fr/hal-01276197

Lavigne, M. (2016). Les faiblesses ludiques et pédagogiques des jeux sérieux. Dans P. Bonfils, P. Dumas, et L. Massou (dir.), Numérique et éducation: Dispositifs, jeux, enjeux, hors-jeux (vol. 34, p. 71-82). France : PUN - Éditions Universitaires de Lorraine.

Legrand, M. (2013). Vigie-Nature : sciences participatives et biodiversité à grande échelle. Cahiers des Amériques latines, (72-73), 65-84.

Marec, J. L. (2002). Situations de communication dans la pratique de recherche: du terrain aux composites. Études de communication. Langages, information, médiations, (25), 15-40. 
MENESR - DGESCO (2015). Instruction relative au déploiement de l'éducation au développement durable dans l'ensemble des écoles et établissements scolaires pour la période 2015-2018.

Merzeau, L., et Mulot, H. (2017). Les communs : levier pour l'enseignement (du) numérique à l'école. Hermès, La Revue, (78), 193-200.

Mœglin, P. (2004). Outils et médias éducatifs : une approche communicationnelle. Grenoble : Presses universitaires de Grenoble.

Mœglin, P. (2015). Quand éduquer devient une industrie. Revue Projet, (345), 62-71.

Mœglin, P. (dir.). (2016). Industrialiser l'éducation : anthologie commentée (1913-2012). Saint-Denis, France : Presses universitaires de Vincennes.

Morin, O., et Simonneaux, L. (2013). Forum et wiki, des environnements collaboratifs pour éduquer au développement durable. Penser l'éducation. 241-256

Muracciole, V. (2009). Design and development of a realtime automation to characterise seed by image analysis (Theses). Université d'Angers.

Olry-Louis, I. (2003). Coopérer et apprendre par le dialogue - enjeux et perspectives. L'orientation scolaire et professionnelle, (32/3), 343-358.

Olry-Louis, I., et Soidet, I. (2003). Coopérer pour co-construire des savoirs : une approche différentielle. L'orientation scolaire et professionnelle, (32/3), 503-535.

Partoune, C. (2006). Un modèle pédagogique global pour une approche du paysage fondée sur les nouvelles technologies de l'information et de la communication (NTIC). L'Information géographique, 70(3), 117-122.

Peraya, D. (2018). Technologies, innovation et niveaux de changement: les technologies peuvent-elles modifier la forme universitaire? Distances et médiations des savoirs. Distance and Mediation of Knowledge, 2018(21). Consulté à l'adresse http://journals.openedition.org/dms/2111

Plantard, P. (2016). Les imaginaires numériques en éducation. Manucius.

Pouliot, C. (2015). Quand les citoyen.ne.s soulèvent la poussière: la controverse autour de la poussière métallique à Limoilou. Carte blanche.

Pruneau, D., Et Jai, B., Khattabi, A., Benbrahim, S., et Langis, J. (2017). La pensée design et Facebook comme outils d'accompagnement pédagogique en résolution de problèmes environnementaux. Info CRDE, (20), 13-14.

Pruneau, D., Kerry, J., Freiman, V., Langis, J., et Cormier, J. (2016). Les rapports préoccupants des futurs enseignants avec les milieux physiques et numériques. Education Relative à l'Environnement : regards, recherches, réflexions, 13(2). Consulté à l'adresse http://www.revue-ere.uqam.ca/categories/PDF/13-1 3.pdf

Sauvé, L. (2008). Un attracteur étrange, une « invasion barbare ». Revue POUR. Dossier: systèmes de formation et développement durable, (198).

Sauvé, L. (2013). Au cœur des questions socio-écologiques: des savoirs à construire, des compétences à développer. Éducation relative à l'environnement : Regards - Recherches - Réflexions, 11, 19-40.

Schmitt, D. (2016). Construction des connaissances : limites et écueils des jeux numériques. Dans P. Bonfils, P. Dumas, et L. Massou (dir.), Numérique et éducation : Dispositifs, jeux, enjeux, hors jeux (Vol. 34, p. 33-44). France : PUN Éditions Universitaires de Lorraine.

Taillard, P. (2017). Vision de l'académie sur la formation des enseignants au et par le numérique. Présenté à Journée d'études. Numérique et nouveaux enjeux de la formation, ESPE de Paris.

Thizeau, V. (2015, mai 7). Six lycéens de Bascan ont fait leur COP 21. Consulté 18 décembre 2018, à l'adresse https://lyc-bascan.fr/six-lyceens-de-bascan-ont-fait-leur-cop-21/

Touir, G. (2015). S'approprier les technologies numériques en milieu associatif au Québec. Dans P. Dias da Silva et A. Alves (dir.), TEM 2015 : Proceedings of the Technology et Emerging Media Track. Ottawa, June 3-5, 2015.

Tricot, A. (2017). L'innovation pédagogique. Paris, France : Éditions Retz.

Zwang, A. (2016). La légitimation d'expositions itinérantes pour l'éducation au développement durable: des objectifs de 1'École à ceux des producteurs. RDST, 13, 21-49.

Zwang, A. (2019). Quand le numérique interroge la culture scolaire. Une étude de cas en éducation au développement durable. Spirale : revue de recherches en éducation, 63(1), 77-88. 
Zwang, A., et Chamboredon, J. (2010). Peut-on évaluer les effets des projets d'ÉDD ? Les cahiers pédagogiques. Dossier «l'éducation au développement durable : comment faire?», (478), 45-47.

Zwang, A., et Girault, Y. (2012). Quelle(s) spécificité(s) pour l'Éducation au Développement Durable? Spirale, (50), 181-195. 